\title{
Matching and Stabilization by the Method of Controlled Lagrangians
}

WP13 16:00

\author{
Anthony M. Bloch ${ }^{1}$ \\ Department of Mathematics \\ University of Michigan \\ Ann Arbor, MI 48109 \\ abloch@math.lsa.umich.edu
}

\author{
Naomi Ehrich Leonard ${ }^{2}$ \\ Dept. of Mechanical and Aerospace Engineering \\ Princeton University \\ Princeton, NJ 08544 \\ naomi@princeton.edu
}

\author{
Jerrold E. Marsden ${ }^{3}$ \\ Control and Dynamical Systems \\ California Institute of Technology 107-81 \\ Pasadena, CA 91125 \\ marsden@cds.caltech.edu
}

\begin{abstract}
We describe a class of mechanical systems for which the "method of controlled Lagrangians" provides a family of control laws that stabilize an unstable (relative) equilibrium. The controlled Lagrangian approach involves making modifications to the Lagrangian for the uncontrolled system such that the Euler-Lagrange equations derived from the modified or "controlled" Lagrangian describe the closed-loop system. For the closed-loop equations to be consistent with available control inputs, the modifications to the Lagrangian must satisfy "matching" conditions. Our matching and stabilizability conditions are constructive; they provide the form of the controlled Lagrangian, the control law and, in some cases, conditions on the control gain(s) to ensure stability. The method is applied to stabilization of an inverted spherical pendulum on a cart and to stabilization of steady rotation of a rigid spacecraft about its unstable intermediate axis using an internal rotor.
\end{abstract}

\section{Introduction}

We present new developments in our constructive approach to the derivation of stabilizing control laws for Lagrangian mechanical systems; we refer to the approach as the method of controlled Lagrangians. The guiding principle behind our methodology is to consider a class of control laws that yield closed-loop dynamics which remain in Lagrangian form. The advan-

\footnotetext{
${ }^{1}$ Research partially supported by the National Science Foundation grants DMS-9496221 and DMS-9803181, AFOSR grant F49620-96-1-0100 and a Guggenheim Fellowship.

${ }^{2}$ Research partially supported by the National Science Foundation under grant BES-9502477 and by the Office of Naval Research under grant N00014-96-1-0052.

${ }^{3}$ Research partially supported by AFOSR Grant F49620-951-0419
}

tage of requiring Lagrangian closed-loop dynamics is that stabilization can be understood in terms of energy, and energy methods provide Lyapunov functions. Being Lyapunov-based, the method yields large and computable basins of stability, which become asymptotically stable when dissipative controls are added.

In this paper, we give sufficient conditions under which our algorithmic approach provides a control law that yields a closed-loop system in Lagrangian form. The Lagrangian for the closed-loop system is called the controlled Lagrangian. We refer to the conditions as matching conditions since they ensure that the EulerLagrange equations derived from the controlled Lagrangian are consistent with available control inputs, i.e., they match the controlled Euler-Lagrange equations for the given mechanical system.

The derived matching conditions define a general class of mechanical systems which includes balance systems, such as inverted pendula, as well as systems with gyroscopic forces such as satellites and underwater vehicles with internal rotors.

Using energy methods we give further sufficient conditions under which the control gain(s) can be selected to ensure stabilization of the unstable (relative) equilibrium of interest.

As we have indicated, the matching and stabilizability conditions are constructive, providing a parametrized family of controlled Lagrangians and control laws. The parameter(s) are the control gain(s) which can be selected for closed-loop stability using the stabilizability condition or, if necessary, more general energy methods.

The method of controlled Lagrangians is described in Bloch, Leonard and Marsden [1997]. The approach generalizes and makes algorithmic the result in Bloch, Krishnaprasad, Marsden and Sánchez de Alvarez [1992] for stabilization of unstable middle axis rotation of a 
rigid spacecraft using a single internal rotor. The basic idea behind our approach was introduced in Bloch, Marsden and Sánchez de Alvarez [1997].

In this paper we restrict to controlled Lagrangians that only involve modifications to the system's kinetic energy. One can also consider modifications to the potential energy for stabilization and tracking purposes. In future work, we will incorporate into our algorithm modifications to the potential energy such as the methods of Van der Schaft [1986] and the addition of symmetry-breaking potentials as in Leonard [1997]. Other relevant work involving energy methods in control and stabilization includes Wang and Krishnaprasad [1992], Koditschek and Rimon [1990], Baillieul [1993], and Åström and Furuta [1996].

This paper is organized as follows. In $\S 2$, we outline the controlled Lagrangian approach to stabilization. In $\S 3$, we address matching and stabilization for certain kinds of mechanical systems which can be treated with a two-step modification to the Lagrangian. The construction is applied to the inverted spherical pendulum on a cart. In $\S 4$, we address matching and stabilization for mechanical systems that have dynamics described by Euler-Poincaré equations. The construction is applied to stabilize steady rotation of a rigid spacecraft about its unstable intermediate axis using an internal rotor. In Bloch, Leonard and Marsden [1997] we discussed the spacecraft in a somewhat ad hoc fashion. Here the controls are derived from the general theory. Proofs, more detailed discussion and additional examples of these results, including stabilization of an underwater vehicle with internal rotors, can be found in Bloch, Leonard and Marsden [1998].

\section{Controlled Lagrangian Approach}

The controlled Lagrangian approach begins with a mechanical system with an uncontrolled (free) Lagrangian equal to kinetic energy minus potential energy. We modify the kinetic energy to produce a new controlled Lagrangian which describes the dynamics of the controlled closed-loop system.

Suppose our system has configuration space $Q$ and that a Lie group $G$ acts freely and properly on $Q$. It is useful to keep in mind the case in which $Q=S \times G$ with $G$ acting only on the second factor by acting on the left by group multiplication.

For example, for the inverted planar pendulum on a cart, $Q=S^{1} \times \mathbb{R}$ with $G=\mathbb{R}$, the group of reals under addition (corresponding to translations of the cart), while for a rigid spacecraft with a rotor, $Q=\mathrm{SO}(3) \times S^{1}$, where now the group is $G=S^{1}$, corresponding to rotations of the rotor.

Our goal is to control the variables lying in the shape space $Q / G$ (in the case in which $Q=S \times G$, then $Q / G=S$ ) using controls that act directly on the variables lying in $G$. We assume that the Lagrangian is invariant under the action of $G$ on $Q$, where the action is on the factor $G$ alone. In many specific examples, such as those given below, the invariance is equivalent to the Lagrangian being cyclic in the $G$-variables. Accordingly, this produces a conservation law for the free system. Our construction will preserve the invariance of the Lagrangian, thus providing us with a modified or controlled conservation law. Throughout this paper we will assume that $G$ is an abelian group.

The essence of the modification of the Lagrangian involves changing the metric tensor $g(\cdot, \cdot)$ that defines the kinetic energy $\frac{1}{2} g(\dot{q}, \dot{q})$.

The tangent space to $Q$ can be split into a sum of horizontal and vertical parts defined as follows: for each tangent vector $v_{q}$ to $Q$ at a point $q \in Q$, we can write a unique decomposition

$$
v_{q}=\operatorname{Hor} v_{q}+\operatorname{Ver} v_{q}
$$

such that the vertical part is tangent to the orbits of the $G$-action and where the horizontal part is the metric orthogonal to the vertical space; that is, it is uniquely defined by requiring the identity

$$
g\left(v_{q}, w_{q}\right)=g\left(\operatorname{Hor} v_{q}, \text { Hor } w_{q}\right)+g\left(\operatorname{Ver} v_{q}, \operatorname{Ver} w_{q}\right)
$$

where $v_{q}$ and $w_{q}$ are arbitrary tangent vectors to $Q$ at the point $q \in Q$. This choice of horizontal space coincides with that given by the mechanical connection; see, for example, Marsden [1992].

For the kinetic energy of our controlled Lagrangian, we use a modified version of the right hand side of equation (2.2). The potential energy remains unchanged. The modification consists of three ingredients:

1. a new choice of horizontal space, denoted $\mathrm{Hor}_{\tau}$,

2. a change $g \rightarrow g_{\sigma}$ of the metric on horizontal vectors and

3. a change $g \rightarrow g_{\rho}$ of the metric on vertical vectors.

Let $\xi_{Q}$ denote the infinitesimal generator corresponding to a Lie algebra element $\xi \in \mathfrak{g}$, where $\mathfrak{g}$ is the Lie algebra of $G$ (see Marsden [1992] or Marsden and Ratiu [1994]). Thus, for each $\xi \in \mathfrak{g}, \xi_{Q}$ is a vector field on the configuration manifold $Q$ and its value at a point $q \in Q$ is denoted $\xi_{Q}(q)$.

Definition 2.1 Let $\tau$ be a Lie algebra valued horizontal one form on $Q$; that is, a one form with values in the Lie algebra $\mathfrak{g}$ of $G$ that annihilates vertical vectors. The $\tau$-horizontal space at $q \in Q$ consists of tangent vectors to $Q$ at $q$ of the form $\operatorname{Hor}_{\tau} v_{q}=\operatorname{Hor} v_{q}-[\tau(v)]_{Q}(q)$, which also defines $v_{q} \mapsto \operatorname{Hor}_{\tau}\left(v_{q}\right)$, the $\tau$-horizontal projection. The $\tau$-vertical projection operator is defined by $\operatorname{Ver}_{\tau}\left(v_{q}\right):=\operatorname{Ver}\left(v_{q}\right)+[\tau(v)]_{Q}(q)$.

Definition 2.2 Given $g_{\sigma}, g_{\rho}$ and $\tau$, we define the controlled Lagrangian to be the following Lagrangian 
which has the form of a modified kinetic energy minus the potential energy $(V)$ :

$$
\begin{aligned}
L_{\tau, \sigma, \rho}(v)= & \frac{1}{2}\left[g_{\sigma}\left(\operatorname{Hor}_{\tau} v_{q}, \operatorname{Hor}_{\tau} v_{q}\right)\right. \\
& \left.+g_{\rho}\left(\operatorname{Ver}_{\tau} v_{q}, \operatorname{Ver}_{\tau} v_{q}\right)\right]-V(q) .
\end{aligned}
$$

The equations corresponding to this Lagrangian will be our closed-loop equations. The new terms appearing in those equations corresponding to the directly controlled variables are interpreted as control inputs. The modifications to the Lagrangian are chosen so that no new terms appear in the equations corresponding to the variables that are not directly controlled. We refer to this process as matching.

Once the control law is derived using the controlled Lagrangian, the closed-loop stability of an equilibrium can be determined by energy methods, using any available freedom in the choice of $\tau, g_{\sigma}$ and $g_{\rho}$.

If we make a couple of assumptions that are not very restrictive on the choice of metric $g_{\sigma}, L_{\tau, \sigma, \rho}(v)$ has the following structure that will be useful for the development.

Theorem 2.3 Assume that $g=g_{\sigma}$ on Hor and Hor and Ver are orthogonal for $g_{\sigma}$. Then

$L_{\tau, \sigma, \rho}(v)=L\left(v+\tau(v)_{Q}\right)+\frac{1}{2} g_{\sigma}\left(\tau(v)_{Q}, \tau(v)_{Q}\right)+\frac{1}{2} \gamma(v)$

where $v \in T_{q} Q$ and $\gamma(v)=\left(g_{\rho}-g\right)\left(\operatorname{Ver}_{\tau}(v), \operatorname{Ver}_{\tau}(v)\right)$.

\section{Mechanical Systems: $L_{\tau, \sigma}$ Case}

In this section we address matching, stabilizability and stabilization of systems in a simplified setting in which we can take $g_{\rho}=g$. The inverted planar pendulum and spherical pendulum on a cart both fit into this setting. In this case, $\gamma=0$ and by Theorem 2.3 the controlled Lagrangian is

$$
L_{\tau, \sigma}(v)=L\left(v+\tau(v)_{Q}\right)+\frac{1}{2} g_{\sigma}\left(\tau(v)_{Q}, \tau(v)_{Q}\right) .
$$

\subsection{Matching and Stabilization}

Locally, we write coordinates for $Q$ as $x^{\alpha}, \theta^{a}$ where $x^{\alpha}, \alpha=1, \ldots n$ are coordinates on the shape space $Q / G$ and where $\theta^{a}, a=1, \ldots, r$ are coordinates for the abelian group $G$. For the uncontrolled system, the variables $\theta^{a}$ will be cyclic coordinates in the classical sense. We write the given Lagrangian in these coordinates (with the summation convention in force) as

$L\left(x^{\alpha}, \dot{x}^{\beta}, \dot{\theta}^{a}\right)=\frac{1}{2} g_{\alpha \beta} \dot{x}^{\alpha} \dot{x}^{\beta}+g_{\alpha a} \dot{x}^{\alpha} \dot{\theta}^{a}+\frac{1}{2} g_{a b} \dot{\theta}^{a} \dot{\theta}^{b}-V\left(x^{\alpha}\right)$.

The conserved quantity, that is the momentum conjugate to the cyclic variable $\theta^{a}$ for the preceding Lagrangian, is given by

$$
J_{a}=\frac{\partial L}{\partial \dot{\theta}^{a}}=g_{\alpha a} \dot{x}^{\alpha}+g_{a b} \dot{\theta}^{b}
$$

The equations of motion for the control system where the controls $u_{a}$ act in the $\theta^{a}$ directions are the controlled Euler-Lagrange equations:

$$
\begin{aligned}
\frac{d}{d t} \frac{\partial L}{\partial \dot{x}^{\alpha}}-\frac{\partial L}{\partial x^{\alpha}} & =0 \\
\frac{d}{d t} \frac{\partial L}{\partial \dot{\theta}^{a}} & =u_{a} .
\end{aligned}
$$

We shall write the given horizontal one form $\tau$ in coordinates as $\tau^{a}=\tau_{\alpha}^{a} d x^{\alpha}$. Thus,

$$
[\tau(v)]_{Q}=\left(0, \tau_{\alpha}^{a} \dot{x}^{\alpha}\right)
$$

Substituting into (3.1) we get

$$
L_{\tau, \sigma}=L\left(x^{\alpha}, \dot{x}^{\alpha}, \dot{\theta}^{a}+\tau_{\alpha}^{a} \dot{x}^{\alpha}\right)+\frac{1}{2} \sigma_{a b} \tau_{\alpha}^{a} \tau_{\beta}^{b} \dot{x}^{\alpha} \dot{x}^{\beta} .
$$

We use the notation $\sigma_{a b}$ for the $a b$ components of $g_{\sigma}$ and, later on, shall likewise use notation $\rho_{a b}$ for the $a b$ components of $g_{\rho}$.

From (3.6) and (3.2) we find that the associated controlled conserved quantity is given by

$$
\begin{aligned}
\tilde{J}_{a}=\frac{\partial L_{\tau, \sigma}}{\partial \dot{\theta}^{a}} & =\frac{\partial L}{\partial \dot{\theta}^{a}}\left(x^{\alpha}, \dot{x}^{\alpha}, \dot{\theta}^{b}+\tau_{\alpha}^{b} \dot{x}^{\alpha}\right) \\
& =g_{\alpha a} \dot{x}^{\alpha}+g_{a b}\left(\dot{\theta}^{b}+\tau_{\alpha}^{b} \dot{x}^{\alpha}\right) .
\end{aligned}
$$

The controlled conserved quantity gives the $\theta$ EulerLagrange equations and this determines the control law, as this is the direction in which we are assuming we have control actuation. For the construction to make sense, however, we need to make sure that the $x$ EulerLagrange equations for $L$ and $L_{\tau, \sigma}$ agree since there is no control actuation in these directions. This latter condition is the matching condition.

Consider the following three assumptions:

Assumption M-1. $\tau_{\alpha}^{b}=-\sigma^{a b} g_{\alpha a}$.

Assumption M-2. $\sigma^{b d}\left(\sigma_{a d, \alpha}+g_{a d, \alpha}\right)=2 g^{b d} g_{a d, \alpha}$.

Assumption M-3. $\tau_{\alpha, \delta}^{b}-\tau_{\delta, \alpha}^{b}-g^{d b} g_{a d, \alpha} \tau_{\delta}^{a}=0$.

Theorem 3.1 Under Assumptions $M-1, M-2, M-3$, the Euler-Lagrange equations for the controlled Lagrangian $L_{\tau, \sigma}$ coincide with the controlled EulerLagrange equations.

For example, if 1) $\sigma_{a b}=\sigma g_{a b}$ for a constant $\sigma$ (this defines $\sigma_{a b}$ ) 2) $g_{a b}$ is independent of $x^{\alpha}$ (a condition on the metric tensor) 3$) \tau_{\alpha}^{b}=-(1 / \sigma) g^{a b} g_{\alpha a}$ (this defines $\tau_{\alpha}^{b}$ ) and 4) $g_{\alpha a, \delta}=g_{\delta a, \alpha}$ (a second condition on the metric), then all three of M-1, M-2 and M-3 hold, so we have matching. The $\sigma$ in this example is a free variable and can be interpreted as the control gain.

The control law is determined from the difference between the conservation laws for the controlled and 
the uncontrolled Lagrangians. In fact, since $\tilde{J}_{a}=J_{a}+$ $g_{a b} \tau_{\alpha}^{b} \dot{x}^{\alpha}$, and since $\tilde{J}_{a}$ is conserved, we may write

$$
u_{a}=\frac{d}{d t} J_{a}=\frac{d}{d t} \tilde{J}_{a}-\frac{d}{d t} g_{a b} \tau_{\alpha}^{b} \dot{x}^{\alpha}=-\frac{d}{d t}\left(g_{a b} \tau_{\alpha}^{b} \dot{x}^{\alpha}\right) .
$$

We can eliminate the accelerations $\ddot{x}^{\alpha}$ by making use of the fact that the Euler-Lagrange equations for $x$ hold (for both $L$ and $L_{\tau, \sigma}$ ).

If the matching conditions are satisfied, we obtain a parametrized family of closed-loop Lagrangian systems. From this we can use energy methods to derive the following sufficient condition for stabilizability. The condition defines the choice of control gain $(\mathrm{s}) \sigma_{a b}$ that ensures stability.

Theorem 3.2 Suppose the conditions for Theorem 3.1 hold (i.e., matching holds with the controlled Lagrangian $L_{T, \sigma}$ ). Then the system is stabilized about a given equilibrium if the second variation of

$$
\frac{1}{2}\left(g_{\alpha \beta}+g_{a \alpha}\left(\sigma^{a b}-g^{a b}\right) g_{b \beta}\right) \dot{x}^{\alpha} \dot{x}^{\beta}+V
$$

evaluated at the equilibrium is definite.

For the proof see Bloch, Leonard and Marsden [1998] which makes use of a controlled Routhian.

\subsection{Inverted Spherical Pendulum}

We apply the above results to the controlled spherical pendulum on a cart in the $x y$-plane. This generalizes the planar pendulum example (see Bloch, Leonard and Marsden [1997]) and provides a nontrivial example of matching and stabilization in the case where we only need a controlled Lagrangian of the form $L_{\tau, \sigma}$. In this case we have independent controls that can move the cart in the $x$ and $y$ directions.

Consider then a spherical pendulum with bob of mass $m$ on a movable base of mass $M$, as in figure 3.1. The base is idealized to be a point (or a symmetric planar body) as this simplifies the calculations without affecting the essential dynamics.

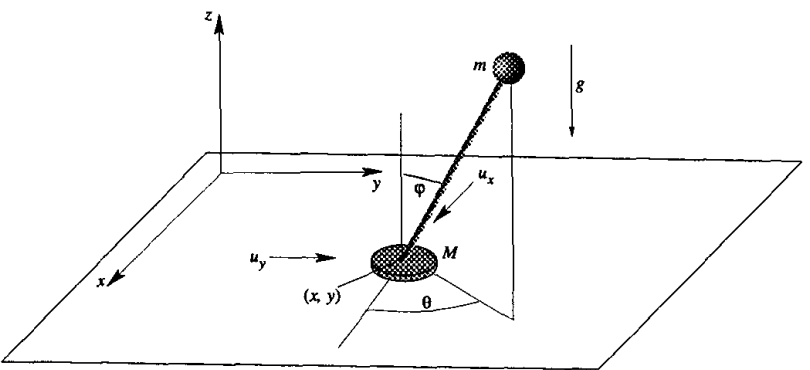

Figure 3.1: The inverted spherical pendulum on a $2 \mathrm{~d}$ cart.

The free Lagrangian for the spherical pendulum on a cart is

$$
L=\frac{1}{2} M\left(\dot{x}^{2}+\dot{y}^{2}\right)+\frac{1}{2} m\left(\dot{x}^{2}+\dot{y}^{2}+r^{2} \dot{\phi}^{2}\right.
$$

$$
\begin{aligned}
& +\quad r^{2} \sin ^{2} \phi \dot{\theta}^{2}+2 r \cos \phi \dot{\phi}(\dot{x} \cos \theta+\dot{y} \sin \theta) \\
& +\quad 2 r \sin \phi \dot{\theta}(-\dot{x} \sin \theta+\dot{y} \cos \theta)) \\
& +\quad m g r(1-\cos \phi)
\end{aligned}
$$

where $\phi$ and $\theta$ are spherical coordinates measured in a frame with origin fixed on the (point) cart, but with orientation that remains fixed with respect to inertial space. The angle $\phi$ represents the deflection from the vertical while $\theta$ represents the angle between the pendulum and the $x$-axis. The controlled equations are the Lagrangian equations with control forces $u_{x}$ and $u_{y}$ in the $x$ and $y$ equations respectively. Note that the Lagrangian is cyclic in $x$ and $y$. However, the system is in fact $S E(2)$ invariant, as one would expect physically.

For the purposes of applying the theory discussed above we choose the symmetry directions to be the $x$ and $y$ directions ignoring for the moment the additional $S^{1}$ symmetry. We shall return to this later.

Note that $g_{a b}$ is constant and $\frac{\partial}{\partial \theta}(\cos \phi \cos \theta)=$ $\frac{\partial}{\partial \phi}(-\sin \phi \sin \theta), \frac{\partial}{\partial \theta}(\cos \phi \sin \theta)=\frac{\partial}{\partial \phi}(\sin \phi \cos \theta)$, i.e., $g_{a \alpha, \delta}=g_{\delta a, \alpha}$ holds. We choose $\sigma_{a b}=\sigma g_{a b}$, where $\sigma$ is a constant, and $\tau_{\alpha}^{b}=-(1 / \sigma) g^{a b} g_{\alpha a}$. Then, Assumptions M-1, M-2 and M-3 all hold and we get matching by Theorem 3.1. In this case, we have

$$
\begin{gathered}
\tau_{\phi}^{x}=-\lambda \cos \phi \cos \theta, \quad \tau_{\theta}^{x}=-\lambda(-\sin \phi \sin \theta) \\
\tau_{\phi}^{y}=-\lambda \cos \phi \sin \theta, \quad \tau_{\theta}^{y}=-\lambda \sin \phi \cos \theta
\end{gathered}
$$

where $\lambda=m r /(\sigma(M+m))$.

Using Theorem 2.3, the controlled Lagrangian is given by the free Lagrangian with velocity shifts

$$
\begin{aligned}
& \dot{x} \rightarrow \dot{x}-\frac{m r}{\sigma(M+m)}(\cos \phi \cos \theta \dot{\phi}-\sin \phi \sin \theta \dot{\theta}) \\
& \dot{y} \rightarrow \dot{y}-\frac{m r}{\sigma(M+m)}(\cos \phi \sin \theta \dot{\phi}+\sin \phi \cos \theta \dot{\theta})
\end{aligned}
$$

and with the addition of the term

$$
\frac{1}{2} \frac{m^{2} r^{2}}{\sigma(M+m)}\left(\cos ^{2} \phi \dot{\phi}^{2}+\sin ^{2} \phi \dot{\theta}^{2}\right) \text {. }
$$

The control laws are given by

$$
\begin{aligned}
& u_{x}=\frac{d}{d t} \frac{m r}{\sigma}(\cos \phi \cos \theta \dot{\phi}-\sin \phi \sin \theta \dot{\theta}) \\
& u_{y}=\frac{d}{d t} \frac{m r}{\sigma}(\cos \phi \sin \theta \dot{\phi}+\sin \phi \cos \theta \dot{\theta})
\end{aligned}
$$

As described above, we can use Lagrange's equations to eliminate the accelerations.

We now use Theorem 3.2 to analyze stability of the pendulum about its upright state, modulo motion in the plane. (Note: stabilization of the cart as well is a goal of ours and will be handled later with the addition of symmetry-breaking potentials). We have

$$
\frac{1}{2}\left(g_{\alpha \beta}+g_{a \alpha}\left(\sigma^{a b}-g^{a b}\right) g_{b \beta}\right) \dot{x}^{\alpha} \dot{x}^{\beta}+V
$$




$$
\begin{aligned}
= & \frac{1}{2} m r^{2}\left\{\left(1+\frac{m}{M+m}\left(\frac{1-\sigma}{\sigma}\right) \cos ^{2} \phi\right) \dot{\phi}^{2}\right. \\
& \left.+\left(1+\frac{m}{M+m}\left(\frac{1-\sigma}{\sigma}\right) \sin ^{2} \phi\right) \dot{\theta}^{2}\right\} \\
& -m g r(1-\cos \phi) .
\end{aligned}
$$

Note that this is independent of $\theta$, reflecting an additional rotational symmetry in this case. Consider the relative equilibrium $\phi=\dot{\phi}=0$. Then, modulo the $\theta$ directions, the second variation of (3.12) with $\dot{\theta}=\mu$ is given by the diagonal matrix with diagonal elements

$$
\begin{aligned}
& d_{1}=m r^{2}\left(1+\frac{m}{M+m}\left(\frac{1-\sigma}{\sigma}\right)\right) \\
& d_{2}=m r^{2}\left(1+\frac{m}{M+m}\left(\frac{1-\sigma}{\sigma}\right)\right) \mu^{2}-m g r .
\end{aligned}
$$

Setting $\sigma=-1 / \kappa$, we have the stability criteria:

- If $\mu=0$, we require $d_{1}<0$, i.e., $\kappa>M / m$,

- If $\mu>0$, we need $d_{1}$ and $d_{2}$ to have the same sign.

\section{Mechanical Systems: Euler-Poincaré Case}

We address the matching problem for the case where the configuration space $Q$ is a nonabelian group $H$ crossed with an abelian group $G$ (a product of tori and lines) and where the Lagrangian is left invariant on $H$, cyclic in the abelian variables and the controls act only on the cyclic variables. The rigid satellite and underwater vehicle with internal rotors fit into this setting.

\subsection{Matching}

Let $L$ denote the left invariant Lagrangian on $T(H \times$ $G)$. Let $l: \mathfrak{h} \times G \rightarrow \mathbb{R}$ be the restriction of $L$ to the identity of $H$ and for a curve $h(t) \in H$ let $\eta(t)=$ $T_{h(t)} L_{h(t)-1} \dot{h}$. Then the (reduced) Lagrangian becomes

$$
l\left(\eta^{\alpha}, \theta^{a}, \dot{\theta}^{b}\right)=\frac{1}{2} g_{\alpha \beta} \eta^{\alpha} \eta^{\beta}+g_{\alpha a} \eta^{\alpha} \dot{\theta}^{a}+\frac{1}{2} g_{a b} \dot{\theta}^{a} \dot{\theta}^{b} .
$$

The conserved quantity, that is the momentum conjugate to the cyclic variable $\theta^{a}$, is given by

$$
J_{a}=\frac{\partial l}{\partial \dot{\theta}^{a}}=g_{a \alpha} \eta^{\alpha}+g_{a b} \dot{\theta}^{b} .
$$

The equations of motion for the control system where the controls $u_{a}$ act in the $\theta^{a}$ directions are the controlled Euler-Poincaré equations:

$$
\begin{aligned}
\frac{d}{d t} \frac{\partial l}{\partial \eta^{\alpha}} & =c_{\alpha \delta}^{\beta} \eta^{\delta} \frac{\partial l}{\partial \eta^{\beta}} \\
\frac{d}{d t} \frac{\partial l}{\partial \dot{\theta}^{a}} & =u_{a} .
\end{aligned}
$$

The formula from Theorem 2.3 gives the controlled Lagrangian in this setting as

$$
\begin{aligned}
& l_{\tau, \sigma, \rho}=l\left(\eta^{\alpha}, \dot{\theta}^{a}+\tau_{\alpha}^{a} \eta^{\alpha}\right)+\frac{1}{2} \sigma_{a b} \tau_{\alpha}^{a} \tau_{\beta}^{b} \eta^{\alpha} \eta^{\beta} \\
& +\frac{1}{2} \gamma_{a b}\left(\dot{\theta}^{a}+g^{a c} g_{c \alpha} \eta^{\alpha}+\tau_{\alpha}^{a} \eta^{\alpha}\right)\left(\dot{\theta}^{b}+g^{b c} g_{c \beta} \eta^{\beta}+\tau_{\beta}^{b} \eta^{\beta}\right)
\end{aligned}
$$

From (4.5) we find that the associated controlled conserved quantity is given by

$$
\tilde{J}_{a}=\frac{\partial l_{\tau, \sigma, \rho}}{\partial \dot{\theta}^{a}}=\rho_{a b}\left(\dot{\theta}^{b}+g^{b c} g_{c \alpha} \eta^{\alpha}+\tau_{\alpha}^{b} \eta^{\alpha}\right) .
$$

Analogous to the three assumptions made in Section 3 for matching, we make two assumptions:

Assumption EP-1. Assume that $\tau_{\alpha}^{a}=-\sigma^{a b} g_{b \alpha}$.

Assumption EP-2. Assume that $\sigma^{a b}+\rho^{a b}=g^{a b}$.

Theorem 4.1 Under the assumptions EP-1 and EP2 the Euler-Poincaré equations for the controlled Lagrangian coincide with the controlled Euler-Poincaré equations.

The control law can be computed by comparing (4.4) to the controlled conservation law. This gives

$$
u_{a}=-\frac{d}{d t}\left(g_{a b} \rho^{b d} \tilde{J}_{d}-J_{a}\right)=g_{a b} \sigma^{b c} g_{c \alpha} \dot{\eta}^{\alpha} .
$$

\subsection{Satellite with Rotor}

Following Krishnaprasad [1985] and Bloch, Krishnaprasad, Marsden and Sánchez de Alvarez [1992], we consider a rigid body with a rotor aligned along the third principal axis of the body. The rotor spins under the influence of a torque $u$ acting on the rotor. The configuration space is $Q=S O(3) \times S^{1}$, with the first factor $H=S O(3)$ being the spacecraft attitude and the second factor $G=S^{1}$ being the rotor angle. The Lagrangian is total kinetic energy of the system, (rigid carrier plus rotor), with no potential energy.

The reduced Lagrangian on $\mathfrak{s o}(3) \times S^{1}$ for this system is

$$
\begin{aligned}
& l(\Omega, \dot{\phi})=\frac{1}{2}\left(\lambda_{1} \Omega_{1}^{2}+\lambda_{2} \Omega_{2}^{2}+I_{3} \Omega_{3}^{2}+J_{3}\left(\Omega_{3}+\dot{\phi}\right)^{2}\right) \\
= & \frac{1}{2}\left[\begin{array}{c}
\Omega_{1} \\
\Omega_{2} \\
\Omega_{3} \\
\dot{\phi}
\end{array}\right]^{T}\left[\begin{array}{cccc}
\lambda_{1} & 0 & 0 & 0 \\
0 & \lambda_{2} & 0 & 0 \\
0 & 0 & \lambda_{3} & J_{3} \\
0 & 0 & J_{3} & J_{3}
\end{array}\right]\left[\begin{array}{c}
\Omega_{1} \\
\Omega_{2} \\
\Omega_{3} \\
\dot{\phi}
\end{array}\right] \quad(4.8)
\end{aligned}
$$

where $\Omega=\left(\Omega_{1}, \Omega_{2}, \Omega_{3}\right)$ is the body angular velocity vector of the carrier, $\phi$ is the relative angle of the rotor, $I_{1}>I_{2}>I_{3}$ are the rigid body moments of inertia, $J_{1}=J_{2}$ and $J_{3}$ are the rotor moments of inertia and $\lambda_{i}=I_{i}+J_{i}$. The momentum conjugate to $\phi$ is

$$
\frac{\partial l}{\partial \dot{\phi}}=l_{3}=J_{3}\left(\Omega_{3}+\dot{\phi}\right) \text {. }
$$

The equations of motion with a control torque $u$ acting on the rotor are

$$
\begin{aligned}
\lambda_{1} \dot{\Omega}_{1} & ={ }_{\jmath} \lambda_{2} \Omega_{2} \Omega_{3}-\left(\lambda_{3} \Omega_{3}+J_{3} \dot{\phi}\right) \Omega_{2} \\
\lambda_{2} \dot{\Omega}_{2} & =-\lambda_{1} \Omega_{1} \Omega_{3}+\left(\lambda_{3} \Omega_{3}+J_{3} \dot{\phi}\right) \Omega_{1} \\
\lambda_{3} \dot{\Omega}_{3}+J_{3} \ddot{\phi} & =\left(\lambda_{1}-\lambda_{2}\right) \Omega_{1} \Omega_{2} \\
\dot{l}_{3} & =u .
\end{aligned}
$$


Next, we form the controlled Lagrangian and apply the Euler-Poincaré matching theorem. Since the abelian group $G=S^{1}$ is one-dimensional, $g_{a b}, \sigma_{a b}$ and $\rho_{a b}$ are all scalars. From (4.8), $g_{a b}=J_{3}$. We let $\sigma_{a b}=\sigma J_{3}$ and $\rho_{a b}=\rho J_{3}$ where $\sigma$ and $\rho$ are dimensionless scalars. For matching we should choose $\tau$ according to Assumption EP-1, i.e.,

$$
\left(\begin{array}{lll}
\tau_{\Omega_{1}}^{\phi} & \tau_{\Omega_{2}}^{\phi} & \tau_{\Omega_{3}}^{\phi}
\end{array}\right)=-\frac{1}{\sigma J_{3}}\left(\begin{array}{lll}
0 & 0 & J_{3}
\end{array}\right) .
$$

Further, to meet Assumption EP-2, $\rho$ should satisfy

$$
\frac{1}{\sigma J_{3}}+\frac{1}{\rho J_{3}}=\frac{1}{J_{3}} \text { or } \rho=\frac{\sigma}{\sigma-1} \text {. }
$$

Substituting into equation (4.5) with these choices, the controlled Lagrangian is given by

$$
\begin{aligned}
l_{\tau, \sigma, \rho} & =\frac{1}{2}\left(\lambda_{1} \Omega_{1}^{2}+\lambda_{2} \Omega_{2}^{2}+I_{3} \Omega_{3}^{2}+\frac{1}{\sigma} J_{3} \Omega_{3}^{2}\right. \\
& \left.+\frac{\sigma}{\sigma-1} J_{3}\left(\Omega_{3}+\dot{\phi}-\frac{1}{\sigma} \Omega_{3}\right)^{2}\right)
\end{aligned}
$$

where $\sigma$ is a free variable and matching is ensured by Theorem 4.1. Using (4.7), the control law is

$$
u=\frac{1}{\sigma} J_{3} \dot{\Omega}_{3}
$$

Defining $k$ by $1 / \sigma=k I_{3} /(1-k) J_{3}$, and using the equations (4.10) to eliminate accelerations, one finds that the feedback control law (4.13) is

$$
u=k\left(\lambda_{1}-\lambda_{2}\right) \Omega_{1} \Omega_{2}
$$

Once one has the problem in Lagrangian and hence Hamiltonian form, one can proceed to use the energyCasimir or energy-momentum method to determine stability.

As in Bloch, Krishnaprasad, Marsden and Sánchez de Alvarez [1992], we consider the case in which our conserved quantity is equal to zero and the equilibrium is $(0, \bar{M}, 0)$ corresponding to steady rotation about the intermediate axis (unstable for the uncontrolled spacecraft). The energy-Casimir method then shows that

Proposition 4.2 For $k>1-J_{3} / \lambda_{2}$, the equilibrium $(0, \bar{M}, 0)$ is nonlinearly stable for the controlled system.

\section{Final Remarks}

One of the advantages of the stabilization scheme in this paper is that it is systematic. Further, the resulting Lyapunov functions used to prove stability provide a means to design additional dissipation terms that will achieve asymptotic stability. Promising preliminary results on asymptotic stability in the context of the method of controlled Lagrangians can be found in
Bloch, Leonard and Marsden [1998]. For example, simulations of the planar nonlinear inverted pendulum on a cart with control derived using our methodology plus dissipation and an additional symmetry-breaking term demonstrate asymptotic stability of both the pendulum and the cart to the origin.

\section{References}

Åström, K.J. and K. Furuta [1996] Swinging up a pendulum by energy control. IFAC, San Francisco 13.

Baillieul, J. [1993] Stable average motions of mechanical systems subject to periodic forcing. Fields Inst. Comm. 1, 1-23.

Bloch, A.M., P.S. Krishnaprasad, J.E. Marsden and G. Sánchez de Alvarez [1992] Stabilization of rigid body dynamics by internal and external torques. Automatica 28, 745-756.

Bloch, A.M., N.E. Leonard and J.E. Marsden [1997] Stabilization of mechanical systems using controlled Lagrangians. Proc. IEEE Conf. Dec. Contr., San Diego, CA, 2356-2361.

Bloch, A.M., N.E. Leonard and J.E. Marsden [1998] Controlled Lagrangians and the stabilization of mechanical systems. Preprint.

Bloch, A.M., J.E. Marsden, G. Sánchez de Alvarez [1997] Stabilization of relative equilibria of mechanical systems with symmetry. Proc. Notre Dame Conf. on Applied Mathematics, Birkhäuser.

Koditschek, D.E. and E. Rimon [1990] Robot navigation functions on manifolds with boundary. $A d v$. in Appl. Math. 11, 412-442.

Krishnaprasad, P.S. [1985] Lie-Poisson structures, dual-spin spacecraft and asymptotic stability, Nonl. Anal. Th. Meth. and Appl. 9, 1011-1035.

Leonard, N.E. [1997] Stabilization of underwater vehicle dynamics with symmetry-breaking potentials, Systems and Control Letters 32, 35-42.

Marsden, J.E. [1992], Lectures on Mechanics London Mathematical Society Lecture note series. 174, Cambridge University Press.

Marsden, J.E. and T.S. Ratiu [1994] Symmetry and Mechanics. Texts in Applied Mathematics, 17, Springer-Verlag.

Van der Schaft, A. J. [1986], Stabilization of Hamiltonian systems, Nonlinear Analysis, Theory, Methods and Applications, 10, 1021-1035.

Wang, L.S. and P.S. Krishnaprasad [1992] Gyroscopic control and stabilization, J. Nonlinear Sci. 2, 367-415. 